\title{
Absorption studies of ring core double clad fiber
}

\author{
W. J. Lai \\ Nanyang Technological University, Temasek Laboratories @ NTU, 50 Nanyang Drive, Singapore
}

\begin{abstract}
We studied the absorption efficiencies of ring doped core DCF (double clad fiber), and found out that the fiber gives excellent results. This will help to better manage the nonlinear effects in the high power working regimes.
\end{abstract}

Keywords-fiber laser; double clad fiber; Ytterbium doped fiber

\section{INTRODUCTION}

The power of fiber lasers has seen rapid increment over the past decades primarily driven a wide range of applications. The reasons for this power advancement are mainly due to fibers' robustness, compactness, thermal and good pumping efficiencies. It is well known that the pump absorption efficiencies of circular inner cladding DCF is low, hence many noncircular inner cladding DCF have been proposed and fabricated. Glas and coworker has reported an M-profiled (or ring core) fiber, with undoped low index core surrounded by high index doped glass [1], and they claimed that the brightness and output power of the fiber can be as high as that of a conventional DCF. Modal behavior and occurrence of selfimages of this ring core fiber are studied both theoretically and experimentally [2-5]. This self-imaging property could be a potential candidate for coherent beam combination of the high power fiber lasers. In this paper, we study the absorption efficiencies of the ring doped core DCF. The results are compared with the commercial available DCF.

\section{THEORETICAL INVESTIGATIONS}

A numerical simulation is developed to analyze the absorption efficiencies in the active core for different DCF geometries. It is done based on the geometrical optics since the dimension of DCF is much larger than the pump wavelength. We choose octagonal inner cladding shape to allow for fair comparisons with commercial DCF; in this case Liekki Yb1200-20/400. Same doping parameters are used for both fibers. Equal effective doped core area, $A_{\text {eff }}$ as defined in (1) is assumed in the analysis, where $r_{2}$ and $r_{1}$ are the outer and inner doped core radii respectively, and $r_{2}-r_{1}$ is the doped core thickness. The $\mathrm{V}$-parameter of this ring core fiber is defined in (2), where $N A_{\text {core }}$ is the numerical aperture of the fiber core and $\lambda_{\mathrm{s}}$ is the signal wavelength, it has to be less than $\pi$ for single mode operation [4].

$$
\begin{aligned}
& A_{\text {eff }}=\pi\left(r_{2}^{2}-r_{1}^{2}\right) \\
& V=\left[2 \pi\left(r_{2}-r_{1}\right) N A_{\text {core }}\right] / \lambda_{s}
\end{aligned}
$$

\section{RESUlTS AND DisCUSSION}

With the same $A_{\text {eff }}, N A_{\text {core }}$, cladding diameter and cladding NA as Liekki Yb1200-20/400; the pump absorption efficiencies with $r_{1}$ of $50 \lambda$ and $100 \lambda$ are shown in Fig. 1 . The insets are the zoom-in section of the curves and the mode profiles of the DCFs. Pump and signal wavelengths are 915 $\mathrm{nm}$ and $1070 \mathrm{~nm}$ respectively.

The ring doped core structure provides a very high scaling capability compared to the conventional DCF. It gives almost full pump absorption and is more than $13 x$ of that in the standard DCF at a length of one meter. From the obtained results, one can conclude that the further the doped position, the higher the scaling factor.

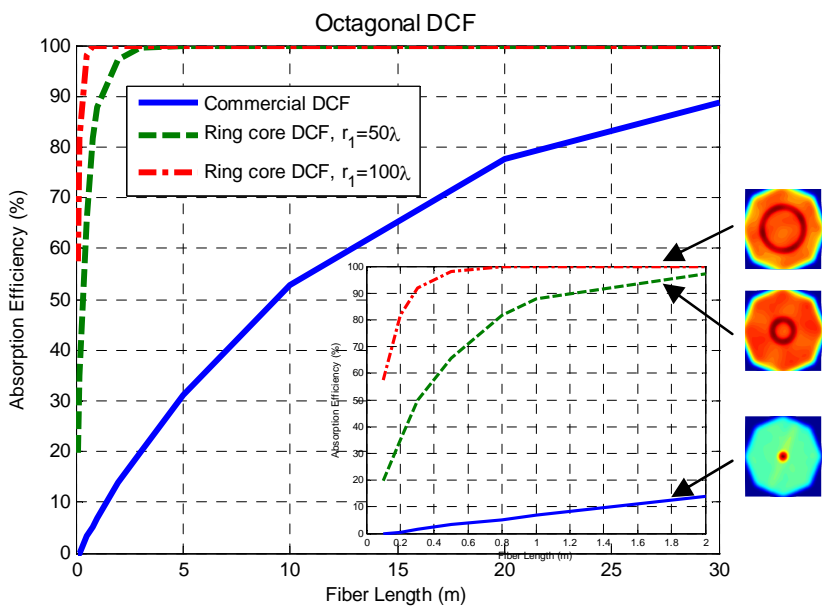

Fig. 1. Absorption efficiencies of ring doped core and conventional DCF

\section{CONCLUSIONS}

We have investigated the absorption efficiencies of the ring doped core DCF, and found out that the fiber gives almost full pump absorption even at short length of fiber. This will help to manage the nonlinear effects of the high power fiber laser or amplifier systems.

\section{REFERENCES}

[1] P. Glas, M. Naumann, and A. Schirrmacher, "A novel design for a high brightness diode pumped fiber laser source," Optics Communications, vol. 122, pp. 163-168, 1996.

[2] P. K. Choudhury and R. A. Lessard, "An estimation of power transmission through a doubly clad optical fiber with an annular core," Microwave and Optical Technology Letters, vol. 29, pp. 402-405, 2001.

[3] M. Hautakorpi and M. Kaivola, "Modal analysis of the self-imaging phenomenon in optical fibers with an annular core," Applied Optics, vol. 45, pp. 6388-6392, 2006.

[4] Y. H. Tsao, D. N. Payne, and W. A. Gambling, "Modal characteristics of three-layered optical fiber waveguides: a modified approach," Journal of Optical Society of America A, vol. 6, pp. 555-563, 1989.

[5] T. Niemeier, R. Ulrich, and S. B. Poole, "Self-imaging by ring-core fibers," in Conference on Optical Fiber Communication and Third International Conference on Optical Fiber Sensors, Washington, D.C., 1985, p. 122. 